\title{
L'HOMME L'Homme
}

182 | avril-juin 2007

Racisme, antiracisme et sociétés

\section{Transmission, identité, corruption}

Réflexions sur trois cas d'hypodescendance

\section{Pierre Savy}

\section{(2) OpenEdition}

\section{Journals}

Édition électronique

URL : https://journals.openedition.org/lhomme/29417

DOI : 10.4000//homme.29417

ISSN : 1953-8103

Éditeur

Éditions de l'EHESS

\section{Édition imprimée}

Date de publication : 2 mai 2007

Pagination : 53-80

ISBN : 978-2-7132-2126-2

ISSN : 0439-4216

Référence électronique

Pierre Savy, «Transmission, identité, corruption », L'Homme [En ligne], 182 | avril-juin 2007, mis en ligne le 01 janvier 2009, consulté le 22 avril 2022. URL : http://journals.openedition.org//homme/29417 ;

DOI : https://doi.org/10.4000/lhomme.29417 
chercher : repérer : avancer

Cet article est disponible en ligne à l'adresse :

http://www.cairn.info/article.php?ID REVUE=LHOM\&ID NUMPUBLIE=LHOM 182\&ID ARTICLE=LHOM 1820053

Transmission, identité, corruption. Réflexions sur trois cas d' hypodescendance

\section{par Pierre SAVY}

\section{| Éditions de l'EHESS | L'Homme}

$2007 / 2-N^{\circ} 182$

ISSN 0439-4216 | ISBN 2-7132-2126-2 | pages 53 à 80

Pour citer cet article :

- Savy P., Transmission, identité, corruption. Réflexions sur trois cas d'hypodescendance, L'Homme 2007/2, № 182, p. $53-80$.

Distribution électronique Cairn pour les Éditions de l'EHESS.

(C) Éditions de l'EHESS. Tous droits réservés pour tous pays.

La reproduction ou représentation de cet article, notamment par photocopie, n'est autorisée que dans les limites des conditions générales d'utilisation du site ou, le cas échéant, des conditions générales de la licence souscrite par votre établissement. Toute autre reproduction ou représentation, en tout ou partie, sous quelque forme et de quelque manière que ce soit, est interdite sauf accord préalable et écrit de l'éditeur, en dehors des cas prévus par la législation en vigueur en France. Il est précisé que son stockage dans une base de données est également interdit. 


\section{Transmission, identité, corruption \\ Réflexions sur trois cas d'hypodescendance}

Pierre Savy

O

N PARLE AUJOURD'HUI VOLONTIERS, dans les sciences sociales, d'une identité plurielle et complexe, que l'individu construirait, au lieu de l'hériter et d'en disposer de façon stable ; on préfere même parfois, à l'idée d'identité, celle d'identification ${ }^{1}$. Le présent travail porte, au contraire, sur la volonté d'imposer à certains individus une identité claire et immuable. L'Occident de tradition germanique au Moyen Âge central, le royaume d'Espagne au début de l'époque moderne, les États-Unis au XX siècle : on peinerait à trouver réalités historiques plus dissemblables. Il n'est pas question d'ignorer les différences et discontinuités majeures qui séparent ces trois réalités et ces trois moments. En chacun, pourtant, fut formulée une théorie de la filiation structurellement identique. Elle illustre, chaque fois, l'application du principe que l'on nomme parfois en français l' "hypodescendance", en calquant le mot d'hypodescent (parfois hypodescendency) en usage en Amérique du Nord. Ce principe est simple : il prévoit que «les enfants d'une union mixte se voient automatiquement assignés au groupe inférieur ${ }^{2}$. C'est de lui qu'il s'agira ici, dans une approche à la fois historique et comparatiste, attentive à montrer comment fonctionna, dans trois contextes différents, ce système de définition de la transmission d'une appartenance et ce qu'il entraîna en termes d'exclusion hors du groupe dominant $^{3}$. Notre objectif est de contribuer à la connaissance du concept d'hypodescendance et au travail de catégorisation de ce système de filiation, et non de faire l'histoire de ces trois moments ni a fortiori de saisir l'histoire de tous les cas d'hypodescendance.

Merci à François Lesueur, Renaud Pasquier, Nicole Savy et David Schreiber pour leurs observations sur l'ensemble du texte, à Bruno Dumézil pour son aide sur le droit barbare, et à Lise Michel pour ses remarques sur la reconnaissance en littérature. Les notes sont renvoyées en fin d'article. 


\section{"Le pire l'emporte"}

Des trois cas étudiés, le premier dans l'ordre du temps est aussi le moins connu. Il s'agit du principe juridique, qui semble trouver son origine dans le droit germanique et qui fut en usage dans l'Occident médiéval, notamment en France, selon lequel «le pire l'emporte». On le rencontre aussi parfois, au gré des sources, sous d'autres formes : «le pire emporte le bon », "le mauvais emporte le bon" ${ }^{4}$. Par ailleurs, on peut rapprocher ces principes dans leur forme française de formules latines se rattachant à la tradition juridique romaine: on trouve dans le Code théodosien une idée proche - et c'est ainsi que l'on lit, dans certaines leçons du Bréviaire d'Alaric interprétant le Code théodosien, que "l'origine va à la personne inférieure " ${ }^{5}$. On lit en outre, chez Isidore de Séville, repris en substance par Raban Maur et Gratien : "L'enfant qui naît prend toujours le statut le plus bas " ${ }^{6}$. Le droit romain n'ignorait nullement cette conception de la filiation.

Ce principe permet de régler la question du statut juridique d'une personne dont une partie de l'ascendance serait libre et une autre partie ne le serait pas: est-elle libre ou non? Non, car «le pire l'emporte ", c'est-àdire que l'ascendance servile prévaut toujours sur l'ascendance libre, que celle-ci soit attestée dans la parenté paternelle ou maternelle. On voit, avec l'usage du mot "pire ", que la hiérarchisation est explicite, ce qui n'est pas exactement le cas dans certains autres exemples que nous évoquerons - les " unions mixtes » dont il est question sont aussi, implicitement ou non, pensées comme des alliances inégales.

Les premières figurations du principe apparaissent dans les anciennes lois barbares: la Lex Ribuaria affirme ainsi que, dans certains cas, «l'on descend toujours vers la condition basse ${ }^{7}$. On lit dans la loi romaine des Burgondes que les enfants nés d'un libre et d'une esclave ou d'un esclave et d'une libre suivent la condition la plus basse ${ }^{8}$. Ce principe d'origine germanique se retrouve parfois dans les mises par écrit du droit coutumier du Moyen Âge tardif. Sur ce point, François Ragueau offre dans son Glossaire, du début du XVII siècle, une fort bonne synthèse. Il note que l'on trouve la formule dans les coutumes du Bourbonnais et dans celles du Nivernais et du Donziais - citons ces dernières, de l'année 1490 :

"Se homme et femme sont conjoincts par mariage, dont l'ung est de condicion servile et l'aultre franc, soit l'homme ou la femme, les enfans qui naistront dudit mariage sont et demourront de la pire condicion, c'est assavoir servile envers le seigneur duquel meut ladicte servitute. Et est ce la coustume desdicts pays de Nivernoys et Donzioys par laquelle l'on dit que le maulvais emporte le bon "?.

Le droit coutumier se distingue, comme toujours, par son extrême diversité - et encore laissons-nous ici de côté l'existence, entre l'homme libre et 
le serf, d'étranges positions intermédiaires de "petite liberté », de «liberté partielle " ou de "demi-liberté " ${ }^{10}$, évidemment impraticables dans les régions où «le pire l'emporte ». Çà et là, diverses pratiques ont prévalu, et il existe des principes contraires à celui que nous considérons - la simple patrilinéarité de la transmission de l'appartenance, pour commencer ; mais un autre principe fréquemment attesté est celui, matrilinéaire, selon lequel "Servitude vient de par les meres ${ }^{11}$. Certains donnent en exemple de proverbe, quelques siècles plus tard : «Il est né d'une mere libre, \& partant il n'est point esclave ${ }^{12}$. Dans les droits romain et canonique, on suit la condition des femmes ${ }^{13}$, dans un sens parfois délibérément favorable à l'enfant (que l'on songe à cette règle romaine «selon laquelle l'enfant naissait ingénu si la mère à un moment quelconque de la gestation avait eu la qualité de libre ${ }^{14}$ ), et l'esclavage de l'époque moderne suit, grossièrement, l'adage "Partus sequitur uentrem " (voir ainsi le cas de la Virginie en $1662 \mathrm{ou}$, pour l'espace français, l'article 13 du Code noir de 1685). Bref, la transmission de la "macule" peut se faire per uentrem ou per uirgam ${ }^{15}$. Thomas d'Aquin, qui rapporte que la loi générale est matrilinéaire (partus sequitur uentrem, encore une fois), sait que certaines contrées font exception, et écrit, en des termes proches de ceux de Gratien :

"Dans certaines terres, qui n'observent pas le droit civil, l'enfant suit la pire condition; ainsi, si le père est serf, bien que la mère soit libre, les enfants seront serfs $[\ldots] \|^{16}$.

On voit qu'il propose une présentation très claire de la situation et de ce qu'elle peut avoir de choquant pour qui est plus habitué à la règle romaine (partus sequitur uentrem). Il semble que celle-ci ne se soit répandue en France que progressivement, et à partir du XIII e siècle seulement, si bien que dans la France médiévale, où des hommes exerçaient une contrainte sur les corps d'une partie de la population, "les enfants issus de ces mariages inégaux naissaient toujours serfs ${ }^{17}$. Le principe peut même s'appliquer à des biens : selon une coutume, en effet, sous certaines conditions, "l'heritage franc devient de la condition du serf ou mortaillable, $\&$ la pire condition attire à soi la meilleure ${ }^{18}$. On voit combien different la solution prévue par le droit germanique, pour lequel «le pire l'emporte», et celle du droit civil.

Tout dépend donc du droit qui prévaut localement. François Ragueau note de surcroît qu'on a pu confondre "père " avec " pire " : cette coquille aurait pu, selon lui, ruiner la patrilinéarité au profit de l'hypodescendance! L'effet démesuré de ce changement de voyelle fait frémir - ou sourire. Dans l'Encyclopédie, un bon siècle après Ragueau, le principe demeure connu, sinon strictement appliqué. On y lit, à l'article " For-mariage »:

"On tient présentement pour maxime, qu'en formariage le pire emporte le bon, c'està-dire que la personne franche, soit la femme ou le mari, qui épouse une personne serve, devient de même condition ${ }^{19}$. 
Notons que l'application de ce principe est ici cantonnée à un cas particulier, celui du formariage, du forismaritagium, c'est-à-dire du mariage d'un serf hors de sa seigneurie d'origine ${ }^{20}$. Le mariage mixte sociologiquement, celui contracté entre serf(ve) et libre, n'est ici envisagé que dans sa dimension de mixité géographique (ou juridictionnelle : en clair, il s'agit de savoir à quelle seigneurie appartiennent les mariés). En outre, l'assignation au groupe inférieur ne concerne pas seulement la descendance, mais même, "horizontalement", celui qui contracte l'union avec la «personne serve» (dans un tout autre contexte, on retrouvera cette prise en considération du conjoint dans la définition de l'appartenance). Un autre exemple, du XIV siècle, le Coutumier bourguignon, présente une position fort nuancée et légèrement différente; d'abord parce qu'il ne considère pas la filiation, mais, une fois encore, le statut du conjoint d'un mariage socialement mixte; ensuite parce qu'il marque une nette réticence à transformer en serf le mari, tandis qu'une altération du statut de la femme paraît moins problématique. Reste que, s'inclinant devant le souci de préserver l'intérêt du seigneur, le Coutumier finit par arriver à une conclusion proche de celles que nous considérons ici :

" homs frans se puet faire serf et advouer seigneur en servitute; mais homs serfs ne puet devenir frans, quelque part qu'il aille, senz faire desavoul au seigneur dessoubz qui il part $»^{21}$.

Et en effet, les Institutes coutumières d'Antoine Loysel indiquent qu' " en formariage, le pire emporte le bon»; les éditeurs précisent que "l'allemand dit presque en mêmes termes: Das Kind folgt der argern Hand (l'enfant suit la pire main)", et rattachent l'application de pareil principe à la loi salique, qui prévoit que "si une personne franche épousait une personne de condition servile, celle des deux qui était franche devenait serve ${ }^{22}$. En revanche, on ne suivrait pas ce principe pour un enfant né d'une union naturelle ou d'une union légitime ${ }^{23}$.

Lointainement, la théorie des quartiers de noblesse a sans doute à voir avec ce principe : nier la pureté de la noblesse à qui a, par exemple sur ses soixante-quatre ancêtres, ne fût-ce qu'un ancêtre roturier, cela ne reviendrait-il pas à appliquer un principe proche de celui selon lequel "le pire l'emporte" ? Bien sûr, on n'exige pas toujours le même nombre de quartiers: "Il faut qu'un Chevalier prouve sa noblesse de quatre Races", rapporte ainsi Antoine Furetière, moins exigeant ${ }^{24}$ (sans compter que la noblesse du père suffit bien souvent à rendre l'enfant noble ${ }^{25}$ ). La noblesse de l'époque moderne défend encore, pour partie du moins, des mythes historiographiques que l'on peut, au risque de l'anachronisme, qualifier d' « ethnicisants ", selon lesquels elle descendrait des Francs, à la différence du peuple $^{26}$. Dans toute l'histoire de la noblesse, le principe héréditaire a 
continué de prévaloir - en 1690 encore, Furetière ne note-t-il pas : "Les vrais Nobles sont les Nobles de race, de sang, d'extraction $"{ }^{27}$ ? Le sang demeure le critère fondateur de la noblesse, idéalement en tout $\operatorname{cas}^{28}$, et quitte à ce que la pratique sociale, par diverses modalités d'ascension dont l'anoblissement est la plus visible, permette la porosité. Ce critère s'appuie sur une vaste réflexion sur le sang, celui, bleu et aristocratique, qui coule dans les veines du noble, et a fortiori celui qui coule dans les veines du roi, ce noble par excellence ${ }^{29}$. Or l'intégrité de cette noblesse passe par l'assurance qu'à aucun moment un mariage mixte n'a été contracté : le pire l'emportant, pareille union entraînerait une irrémédiable dévaluation du lignage.

On peut proposer le schéma suivant, dans lequel, comme dans tous les schémas de parenté figurant dans le présent travail, Ego, par convention, se trouve tout à fait en bas du schéma; en haut à gauche d'Ego, figure la mère; et en haut à droite d'Ego, figure le père, et ainsi de suite, à gauche les femmes et à droite les hommes :

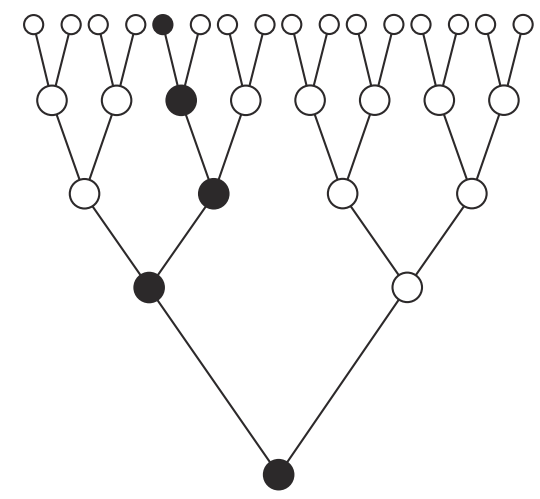

Fig. I L'hypodescendance (“Le pire l'emporte”, Limpieza de sangre, One-drop rule)

On voit qu’à chacun des quatre sauts générationnels représentés, l'appartenance servile, figurée en noir $(\bullet)$, l'a emporté : ainsi Ego n'est-il pas libre car sa mère ne l'était pas; celle-ci ne l'était pas car son père ne l'était pas; celui-ci avait hérité son statut servile de sa propre mère, laquelle le tenait aussi de sa mère, etc. (c'est évidemment de façon arbitraire que l'on a choisi cette ligne de transmission plutôt qu'une autre et, pour des raisons graphiques, on s'en est tenu à la représentation de cinq générations et quatre sauts générationnels). En théorie, un seul ancêtre non libre sur seize suffit, dans ce cas, à rendre Ego non libre. Il est sûr que «Le pire l'emporte » est un principe tourné vers l'ascendance immédiate, afin de déterminer rapidement le statut juridique d'une personne, et peu soucieux 
d'établir une généalogie: notre schéma, valide théoriquement, n’eut sans doute guère d'application pratique. Il reste efficace pour décrire ce principe ancien d'hypodescendance.

\section{La limpieza de sangre}

La "pureté de sang", en espagnol la limpieza de sangre: c'est ainsi que l'on appelle un principe fameux, inventé dans l'Espagne du XV siècle. Certes, des idées proches existaient avant cette période. Suétone ne rapporte-t-il pas qu'Auguste répugna à concéder avec générosité citoyenneté et liberté, de peur que «le sang étranger et servile " ne vînt souiller le sang romain ${ }^{30}$ ? Mais, en Espagne, cette idée prit des formes toute particulières. Rappelons que, le 31 mars 1492, y fut signé le fameux décret d'expulsion des Juifs; cinq ans plus tard, en 1497, le Portugal aussi procéda à leur expulsion. Depuis lors, vécurent dans la Péninsule des "nouveaux-chrétiens ", ou conversos, c'est-à-dire d'anciens Juifs convertis et leurs descendants, parfois devenus sincèrement chrétiens, ainsi que des hommes vivant leur judaïsme en secret, appelés, eux, crypto-Juifs ou marranes (marranos).

Ces catégories posaient manifestement un problème aux pouvoirs religieux chrétiens. Dès avant l'expulsion, ils apportèrent à ce problème une solution inouïe, et contraire à la logique «confessionnelle» et orthodoxe : la limpieza de sangre. C'est en 1449, à Tolède, que furent pris pour la première fois des statuts de limpieza. Il s'agissait, avant de confier des charges publiques ou ecclésiastiques à un individu, de s'assurer de sa chrétienté, et, pour cela, de vérifier, non pas qu'il était baptisé ou observant, mais que son sang était "pur» de tout judaïsme (ou de toute origine maure) - en clair, qu'il n'avait pas un seul ancêtre juif ou maure qui rendît son sang impur ${ }^{31}$. Bref, il s'agissait d'empêcher "les Chrétiens d'ascendance juive " "de jouer un rôle de premier plan dans la société chrétienne de l'Espagne » ${ }^{32}$. Les "Morisques», chrétiens d'origine musulmane ou descendants de musulmans, furent bientôt concernés eux aussi, jusqu’à leur expulsion en 1609.

Notons toutefois que ces statuts étaient pris au coup par coup, dans des institutions supérieures comme les grands collèges, les ordres militaires ou les chapitres ecclésiastiques : ils ne constituaient pas une législation royale ni un dispositif normatif valide à l'échelle de tout le royaume, quoique la Couronne les approuvât ${ }^{33}$. Et, parce qu'ils limitaient l'accès à ces institutions supérieures, ils concernaient, plus que la société tout entière, les élites de la Péninsule ibérique. De plus, parmi les exigences formulées à destination des impétrants, la pureté du sang n'était pas la seule ni même la plus 
importante - la noblesse et la richesse occupaient sans doute une place plus grande (mais la première n'était pas sans rapport avec la notion de pureté). En outre, certains auteurs proposaient une conception complexe des statuts sociaux : à la fin du XV ${ }^{e}$ siècle, dans son Nobiliario vero, Ferrand Mexía défend ainsi l'idée d'une possibilité de purifier son sang en adoptant un comportement honorable, en servant et en combattant, et ce sur plusieurs générations. On pourrait donc "récupérer" la noblesse, qualité ontologique de l'homme perdue avec le péché originel ${ }^{34}$. La limpieza est alors à entendre comme une démarche, non comme un état: on peut laver son sang, et le sang lave (n'y lave-t-on pas l'honneur ?) ${ }^{35}$.

Reste que, si on les considère de manière "structurelle", les statuts de pureté ont cette rigueur extrême. Le schéma pour rendre compte de la " tache » juive faisant perdre sa pureté au sang est rigoureusement le même que celui décrivant "Le pire l'emporte ( figure 1). Et, à la différence de cette maxime germanique, les "statuts de pureté " conduisirent à des enquêtes généalogiques: la validité de notre schéma n'est plus, ici, cantonnée au plan théorique. On le voit, par cette législation discriminante érigée à l'encontre des conversos, était visé le groupe des «nouveauxchrétiens " dans son ensemble, et l'on renonçait à distinguer parmi eux les catholiques sincères des crypto-Juifs. On pouvait donc, dans l'Espagne du $\mathrm{XVI}^{\mathrm{e}}$ siècle, être chrétien de cœur, de pratique, de conviction et aux yeux des Juifs, et être pourtant considéré comme juif en raison d'un défaut de limpieza. La qualification de "juif» est ici à prendre dans le sens non confessionnel et très englobant que lui donnaient les contemporains. Elle désigne des positions qui vont du "nouveau-chrétien », d'ascendance juive plus ou moins lointaine et plus ou moins significative, au Juif déclaré, bien sûr, en passant par le crypto-Juif, le marrane.

Que compter un ascendant juif, aussi éloigné soit-il, devienne comme un stigmate indélébile, voilà qui révèle l'existence d'un état d'esprit qu'il faut bien qualifier de raciste, ce «racisme» n'eût-il pas la possibilité de s'appuyer sur des théories pseudo-scientifiques comme pourront le faire, quelques siècles plus tard, les théories racistes de Gobineau ou des nazis. Entendre la race comme définie scientifiquement, avec des gènes et un patrimoine, est limitatif. Il ne faut pas oublier qu'il existe à l'époque moderne l'idée de dispositions innées immuables propres à un "peuple" particulier ${ }^{36}$, donc du racialisme ante litteram. Les statuts de pureté suscitèrent de nombreuses controverses et l'opposition de certains, au nom de l'esprit évangélique; il n'en est pas moins vrai qu'ils marquent profondément l'Espagne du XVI et du XVII ${ }^{\mathrm{e}}$ siècle, en dépit des efforts parfois consentis pour réduire leur place toujours croissante, par exemple avec la Pragmática de Philippe IV, en 1623. 


\section{La one-drop rule}

Le troisième cas d'hypodescendance que nous voudrions évoquer est la one-drop rule: la règle, également appelée parfois one black ancestor rule ou traceable amount rule, selon laquelle une goutte (one drop) de sang noir suffit à faire qu'un individu soit considéré comme noir. Cette règle avait été théorisée, au début de XXe siècle, dans les travaux de Madison Grant, qui l'appliquait aux enfants nés de l'union d'un Blanc avec un Indien, un Noir, un Hindou, et précisait même que l'enfant né de l'union d'un homme de race européenne avec une Juive ou d'une femme de race européenne avec un Juif était juif ${ }^{37}$. Mais, dans les faits, cette règle était déjà appliquée dans la période esclavagiste de l'histoire américaine et, venue du Sud, valait globalement pour l'ensemble du pays ${ }^{38}$. La catégorie de métis (mulattoes) apparut dans les recensements en 1850 ; mais nombreuses furent alors les oppositions à l'établissement d'une nouvelle catégorie raciale, car l'hypodescendance préservait mieux la pureté du sang blanc $^{39}$. De toute façon, pour certains, "les mulattoes [constituaient] un sous-ensemble de la population noire"; et la catégorie disparut de nouveau du recensement après 1920, «du fait de l'intériorisation de la one drop rule par les blancs comme par les noirs " ${ }^{40}$. Dans les années 1930, l'eugéniste Walter Plecker poursuivit cette réflexion raciste et obtint que la one-drop rule eût force de loi ; elle fut en vigueur aux États-Unis d'Amérique jusqu'à ce que, en 1967, la Cour suprême invalidât le Virginia Racial Integrity Act de Plecker. En d'autres termes, couleur " phénoménologique " (de quelle couleur est visiblement la peau d'un homme) et "appartenance raciale " (à quelle "race » on considère qu'appartient un homme) étaient disjointes, grâce à une construction mentale étrange dont l'un des principaux avantages était, au début, la résolution de la contradiction entre esclavage et démocratie ${ }^{41}$. Une fois encore, la figure 1 décrit bien la one-drop rule.

Des trois cas ici envisagés, cette règle est la plus connue, ainsi que la plus déterminante aujourd'hui encore car, bien qu'abandonnée dans la législation, elle reste forte dans les représentations communes, même si elle paraît reculer : on se rappelle peut-être le refus signifié en 1997 par le joueur de golf Tiger Woods d'être qualifié de joueur "noir » (il se définissait comme Cablinasian, pour "Caucasian-Black-Indian-Asian", "blanc, noir, indien et asiatique»). Cette déclaration eut des conséquences : le pays connut alors un vrai débat sur ce thème, et le Congrès entreprit même une réflexion sur la manière dont on mesurait la race et l' "ethnicité "42. De même, dans le recensement décennal réalisé aux États-Unis, où la question de l'appartenance "raciale" est posée, la proportion d'enfants métis 
qualifiés de "noirs" est passée de $62 \%$ en 1990 à $31 \%$ en 2000 . Reste que l'assignation au groupe est souvent faite selon le principe de l'hypodescendance, quoiqu'il n'y ait pas, officiellement, de qualification négative du groupe "noir». Le principe est même ambigu, car c'est aussi pour l'affirmative action (la "discrimination positive») que les pouvoirs américains comptent les Noirs, si bien que certaines associations noires tiennent à cette pratique et qu'il arrive - la littérature juridique a rendu célèbres ces multiples cas - qu'un homme, blanc d'apparence, invoque une ascendance noire pour réclamer des droits fédéraux ou l'intervention de l'Equal Employment Opportunity Commission (EEOC) ${ }^{43}$. Dans la littérature anthropologique et sociologique, le débat a été posé comme une opposition entre assignation par la couleur (la "vraie » couleur de la peau) et hypodescendance. La couleur, elle, offre mille nuances: entre noir et blanc, "métis " est le terme général, mais on connaît et l'on a pu utiliser les notions de mulâtre, de câpre, de jambos, de quarteron, d'octavon, etc. ${ }^{44}$, tandis que l'hypodescendance ignore le métissage - c'est la pratique $\mathrm{du}$ « tout ou rien » en matière raciale, la « réduction binaire d'une extrême diversité phénotypique " ${ }^{45}$. La société brésilienne a adopté une solution toute différente de celle en vigueur aux États-Unis, puisque, au Brésil, les modalités de l'appartenance ne sont pas binaires.

Ces trois règles d'hypodescendance, proches et distinctes, étant présentées de manière analytique, il paraît utile de réfléchir à présent aux modalités de la transmission de l'appartenance dans d'autres contextes.

\section{D'autres filiations}

On ne peut dénombrer les systèmes de filiation et de transmission, les manières dont, dans des situations données, des sociétés ont pu prévoir comment serait ou non transmise une "appartenance». On ne peut présenter, fût-ce abstraitement, les autres solutions possibles pour déterminer à quel groupe appartient un enfant dont la situation est incertaine. On le voit, l'union mixte, ou, plus généralement, le métissage (la miscegenation anglo-saxonne) occupe une place centrale, car c'est avec ce phénomène que la question de l'appartenance transmise se pose réellement. Un travail qui mettrait en série les systèmes retenus, et qui prendrait en considération d'autres systèmes de transmission encore, par exemple dans les relations entre Turcs et Arméniens, ou entre Hutus et Tutsis, ou encore des civilisations comme celle des Grecs par rapport aux barbares, serait passionnant.

Présentons toutefois quelques systèmes alternatifs. La filiation unilinéaire, c'est-à-dire l'assignation au groupe en fonction de l'appartenance de l'un des parents, soit le père, soit la mère, est sans doute la solution la plus 
souvent adoptée : patrilinéarité et matrilinéarité, déjà rencontrées quand il s'agissait de l'idée que «le pire l'emporte », sont plus fréquentes que l'hypodescendance et sa symétrique, l'hyperdescendance (nous reparlerons de ce concept). La transmission de l'appartenance juive, telle qu'elle est définie par les Juifs eux-mêmes et telle, au contraire, qu'elle a été définie, au XXe siècle, par un certain nombre de théories et de législations antisémites, semble un autre point de comparaison intéressant pour mieux cerner le fonctionnement et la signification de l'hypodescendance, d'autant que faire l'impasse sur ce point serait laisser dans l'ombre un aspect qui constitue souvent l'arrière-fond du problème de l'hypodescendance, du moins dans le cas de la limpieza de sangre (tandis que, d'une part, l'appartenance à la servitude n'a pas été, à notre connaissance, théorisée par les serfs, dont la "voix " demeure, en dépit des efforts de l'historiographie récente ${ }^{46}$, bien difficilement audible; et que, d'autre part, pour ce qui est de la définition des Noirs par les Noirs, les positions sont moins tranchées).

$\mathrm{Si}$, théoriquement, et sous réserve de preuves apportées par des témoins, un seul ancêtre, même très éloigné, peut suffire à rendre juif un descendant, encore faut-il qu'il y ait continuité absolue entre lui et son descendant par le lien matrilinéaire : en clair, si on se situe après le don de la Torah (avant lui, en effet, il semble que la patrilinéarité primât), voire après l'époque de la mise par écrit de la Michna, et si on laisse de côté le cas de la conversion ainsi que les modifications proposées par les courants libéraux et réformés du judaïsme, il faut que cet ancêtre, ce soit la mère de la mère (etc.) de la mère d'Ego. Affirmation qui n'empêche pas tout à fait le judaïsme de donner à la patrilinéarité son importance, comme le montrent notamment les critères de définition de l'appartenance tribale, aujourd'hui inopérants, ou ceux (qui, eux, sont encore valides) de l'appartenance aux groupes des cohanim et des leviim $^{47}$ : appartient en effet à ces groupes toute personne dont la mère est juive et dont le père appartenait lui-même à pareil groupe. La matrilinéarité demeure requise (pour l'appartenance au peuple juif), redoublée d'une exigence patrilatérale. Dans le judaïsme, on observe donc une filiation bilinéaire, en ce sens que la transmission de l'appartenance au peuple est matrilinéaire et la parenté patrilinéaire (mais conditionnée par l'appartenance au peuple) ${ }^{48}$. La transmission par ligne maternelle ne s'observe évidemment pas seulement dans le judaïsme. L'existence de systèmes matrilinéaires, connus grâce à des travaux d'anthropologues, est attestée dans plusieurs sociétés: aux îles Trobriand bien sûr ${ }^{49}$, ainsi qu'au Cameroun, au Soudan, aux Comores, en Inde, à Sumatra, etc. Certaines sont connues depuis longtemps. Antoine Furetière, encore lui, notait ainsi : 
«En la coste de Malabar, les enfans ne peuvent estre Nobles que du costé de leur mere, parce qu'il leur est permis de prendre autant de maris qu'il leur plaist $\&$ de les quitter, quand bon leur semble ${ }^{50}$.

Peu importe ici l'historicité douteuse de cette noblesse de Malabar ainsi décrite : le principe est légitimé par un autre principe, celui de la certitude du ventre de la mère. La règle romaine Venter matris certus est, ou Mater semper certa est, ne semble pas loin, à laquelle il arrive que l'on rattache aussi la matrilinéarité juive, du reste (bien que, comme l'explique Rabbi Siméon, ce principe doive plutôt se déduire d'un passage du Deutéronome sans rapport avec la «certitude du ventre $\left.{ }^{51}\right)$.

Le schéma est le suivant :

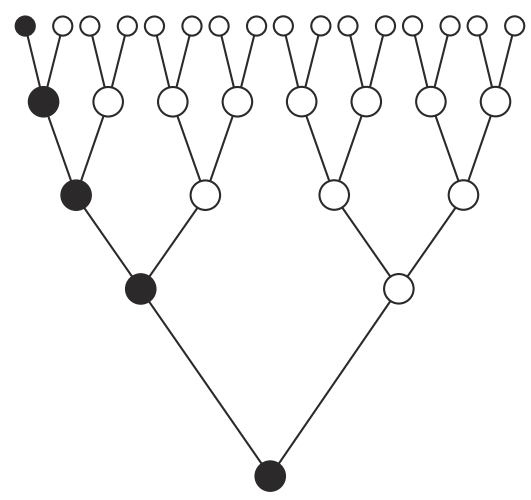

Fig. 2 La matrilinéarité (judéité, noblesse de Malabar, îles Trobriand, etc.)

Ego est juif ou noble de Malabar ou membre d'un groupe social donné (ici colorié de noir, $\bullet$ ) parce que la mère de la mère de la mère de sa mère l'était: tous les autres ancêtres seraient-ils d'un autre peuple (ou d'une autre confession, ou d'un autre groupe social), qu'Ego n'en serait pas moins, à strictement parler, et en théorie, juif selon la loi juive, ou noble à Malabar selon Furetière : bref, membre du même groupe social que cette aïeule de sa parenté maternelle. (Il est évident qu'un schéma décrivant la patrilinéarité serait parfaitement symétrique à celui-ci.)

On voit que l'on n'est pas ici face à un principe comparable à l'hypodescendance : il ne s'agit pas d'un principe, disons, d'hyperdescendance au sens qu'aurait ce mot s'il existait, parce qu'il n'y a pas assignation mécanique à l'un des deux groupes en vertu d'une prédominance du principe bas (ou haut) - sans compter que ce ne serait pas des individus "automatiquement assignés au groupe supérieur ", pour paraphraser la définition de l'hypodescendance, puisque les textes de la tradition juive n'indiquent 
pas que le peuple juif soit «supérieur » aux autres (notons toutefois que les judaïsmes non orthodoxes, en envisageant aussi l'ascendance juive patrilatérale, se rapprochent en fait de ce système de filiation, ce qui ne change rien au caractère non "hiérarchisant ", dans un sens ou dans l'autre, de l'assignation). Dans l'ensemble, il est faux de dire qu'une goutte quelconque de «sang juif» ou de "sang noble de Malabar " rend juif ou noble de Malabar. Et, dans le cas du judaïsme, il n'est pas question de sang ${ }^{52}$.

En dernière analyse, tient-on avec la patrilinéarité (ou matrilinéarité) et l'hypodescendance (ou hyperdescendance) les deux seules manières de déterminer l'identité (entendue comme appartenance à un groupe) ? Bien sûr que non : adoption, calcul des "pourcentages ", incorporation par le mariage, substitution du sol au sang, libre choix de l'individu, prise en considération de la pratique sociale et/ou politique effective sont d'autres solutions possibles, qui pourraient enrichir et complexifier encore le tableau.

\section{Le racisme du $X X^{e}$ siècle}

Les cibles des théories racistes du $\mathrm{XX}^{\mathrm{e}}$ siècle different de celles de la limpieza de sangre: le plus souvent, on n'observe pas, dans la théorie raciale contemporaine, la même radicalité, ou, mieux, la même exigence d'une définition binaire de l'appartenance - sens de la nuance paradoxal, et qui ne s'est évidemment pas montré incompatible avec une violence d'une autre nature et d'une autre ampleur. Les théoriciens de la race du III ${ }^{e}$ Reich se démenèrent avec des notions fragiles et parfaitement racistes, comme celles de "quart-Juifs", de "demi-Juifs» et d'Aryens d'origine juive (Mischlinge de premier degré, de deuxième degré), l'« origine » de ces individus étant parfois exprimée en pourcentages. Il arriva même que certains de ces hommes d'origine juive servissent dans l'armée allemande ${ }^{53}$. Divers textes législatifs furent rédigés : d'abord, le 11 avril 1933, un règlement qui définissait comme «non-aryen » quiconque avait dans les deux générations antérieures un ascendant juif (père, mère, grand-père ou grand-mère), en adoptant comme critère de définition de l'ascendant en question un critère « religieux ", et non « racial ». Il ne s'agissait pas d'hypodescendance stricte, car deux générations seulement étaient concernées ${ }^{54}$. On produisit ensuite des textes plus aboutis: les lois de Nuremberg et un décret de 1935 définissaient qui était aryen et qui était juif. Bernhard Lösener, expert en "questions juives ", est l'artisan du décret qui porte son nom ; dans sa typologie, les «non-Aryens » de 1933 sont remplacés par des « Juifs». Ce décret crée deux groupes de "non-Aryens »: les métis (Mischlinge), d'une part, et les Juifs intégraux ou considérés comme tels, d'autre part. Est juif celui qui a trois grands-parents juifs; ou qui en a deux et, soit appartient à la 
communauté religieuse juive, soit a épousé un Juif ou une Juive, soit est né d'un mariage avec un époux « juif intégral » ou aux trois quarts, soit est né d'une union illégitime dont un partenaire était juif ${ }^{55}$.

Forte de l'expérience nazie, l'Italie fasciste ne resta pas bien longtemps en reste : en 1938, y furent promulguées les « lois raciales ", qui constituaient le prélude à la mise à l'écart des Juifs ${ }^{56}$. Quant à la France de Vichy, le mécanisme y est assez similaire : aux termes d'une première loi, expéditive, est définie comme juive " toute personne issue de trois grands-parents de race juive ou de deux grands-parents de la même race, si son conjoint lui-même est juif ${ }^{57}$, mais, dès 1941, la définition est retravaillée et précisée. La " race " se voit définie de fait par la religion du "grand-parent [sic] ", au prix d'une étonnante distorsion entre les générations et entre les notions de race et de religion : "Est regardé comme étant de race juive le grand-parent ayant appartenu à la religion juive " 58 (c'est nous qui soulignons). Ces lois, comme les lois allemandes, étaient dans l'obligation de définir précisément les critères d'appartenance à telle ou telle " race » : quels individus appartenaient à la "race juive ", et quels individus appartenaient à la "race " italienne ou française. La solution retenue paraît plus binaire que la solution allemande, puisque ces lois ne créaient pas de catégorie de "métis".

Ces choses-là sont bien connues. Le racisme, s'il est cohérent et exigeant, se trouve confronté à des problèmes parfaitement insolubles. C'est ce que montrent les schémas établissant qui est aryen mais devant, dans cette société déjà métissée, rejeter l'hypodescendance stricte pour accepter de considérer comme aryens des Allemands d'origine juive ${ }^{59}$. Ainsi, l'Ego représenté dans la figure 2, qui est un cas-limite, n'aurait pas été considéré comme juif par les nazis, sauf s'il pratiquait la religion juive, tandis que certains que les nazis qualifiaient de Juifs n'auraient pas été reconnus comme tels par la loi juive. Bref, des Juifs sont définis comme non juifs par les nazis, et inversement. En pratique, il n'est pas même sûr que le principe en vigueur revînt à considérer comme juives un nombre de personnes bien supérieur à ce qu'il aurait été selon la loi juive; en tout cas, il ne se contentait pas forcément d'une matrilinéarité. Loin de nous l'idée de donner à la théorie raciale du nazisme une cohérence et une constance qu'elle n'eut pas ; marquée par l'héritage chrétien, elle fut aussi influencée par Gobineau, Darwin ou Chamberlain, mise en ouvre par Darré et d'autres, bref, elle se révèle assez hétéroclite.

Nous n'ignorons pas que l'exigence d'une ascendance purement aryenne fut aussi formulée par les nazis: une sorte de limpieza de sangre, en somme, qui se contentait le plus souvent de remonter jusqu'à 1800 , mais que l'on voulut parfois faire remonter jusqu'à 1750 , voire plus haut encore, jusqu'au XVII siècle et aux plus anciens registres conservés (car il 
est très difficile de remonter plus haut que la guerre de Trente Ans, qui s'achève en 1648). Cette exigence d'une origine familiale "pure ", apparentée à l'hypodescendance, ne concernait pas la société tout entière, mais seulement cette ébauche de caste supérieure que représentait la Schutzstaffel (SS) selon le projet de Heinrich Himmler, définie par des critères raciaux particulièrement exigeants. Du reste, comme la limpieza, cette aryanité visait à protéger la pureté d'une quasi-caste, donc à exclure (en empêchant le mariage avec une personne présentant dans ses origines un défaut d'aryanité), mais pas, comme telle, à causer la mort ${ }^{60}$.

Les nazis traçaient donc d'étranges schémas, et perdaient manifestement leur latin. Leur définition du Juif différait de celle en usage dans le système de l'hypodescendance espagnole, assurément; mais, dans les deux cas, on trouve la même inquiétude, utilisée comme justification de la sévérité des lois, face au risque d'une dégénérescence de l'ensemble de la société. La contagion du "sang» (et aussi, dans certaines sources, d'autres substances corporelles, comme la semence ou le lait ${ }^{61}$ ) entraînerait avec elle la transmission des valeurs et des traits moraux juifs - d'où peut-être la curieuse prise en considération du conjoint dans le décret Lösener ou les lois de Vichy, définissant comme juif l'individu, né lui-même d'une union mixte, et qui aurait épousé un Juif ou une Juive (serait-on juif par sa femme ou son mari ? On se rappelle que celui qui contractait l'union avec un serf pouvait le devenir à son tour ; et l'on sait qu'il existe des exemples de filiation substitutive). Cette peur de la décadence biologique s'est exprimée de façon éclatante dans la politique eugéniste mise en ouvre par l'Allemagne nazie ${ }^{62}$.

Une précision est ici nécessaire : par la loi du Retour, cette loi votée en 1950 pour permettre à tout Juif d'immigrer en Israël, l'État juif admet une appartenance aux critères plus souples, fondée aussi par la persécution - et s'apparentant d'une certaine manière à de l'hyperdescendance, à l'instar des critères déjà évoqués de définition de la judéité selon les conceptions non orthodoxes $^{63}$. Mais le rabbinat orthodoxe, refusant que ce soient les persécuteurs qui dictent l'appartenance à Israël, maintient des principes différents.

\section{L'hypothèse d'une "hyperdescendance"}

On a considéré presque uniquement les cas où le groupe auquel on considère qu'appartient l'individu était jugé négatif; mais, théoriquement, rien n'exclut a contrario l'assignation au groupe supérieur. Il suffirait alors, pour appartenir au groupe en question, de disposer, parmi un nombre considérable d'ancêtres, d'un seul ancêtre ayant appartenu à ce groupe. Empiriquement, le cas est rare; mais il est des situations où l'on peut, avec le même type de "preuves ", établir l'appartenance à un groupe jugé attractif. 
N'est-ce pas ce qui se produit quand, en une forme extrême de reconnaissance, au sens qu'Aristote donne à ce mot dans sa Poétique ${ }^{64}$, deux inconnus, après avoir un peu " fait connaissance » et parlé de leurs familles respectives, tombent dans les bras l'un de l'autre en s'écriant: " Mais alors, nous sommes parents!»? Dans la littérature, pareilles scènes mettent le plus souvent face-à-face deux personnes qui se connaissaient déjà et se reconnaissent - l'Edipe roi de Sophocle ou l'Iphigénie en Tauride d'Euripide constituant des cas archétypaux. Mais nous voulons parler ici d'individus qui ne se connaissaient pas, ou qui, se connaissant, ignoraient qu'ils étaient parents, et qui se découvrent tels. Dans Le Fils naturel, Diderot met en scène une reconnaissance de ce type : à la fin du drame, Lysimond, le père de Rosalie, révèle qu'il est aussi celui de Dorval, le « fils naturel » du titre $^{65}$. Bien sûr, ces reconnaissances semblent improbables, et elles nous emmènent loin de l'hypodescendance; signalons toutefois que certaines œuvres littéraires comme certains textes critiques mettent en avant l'idée d'une " voix du sang ", d'un " cri du sang " ou d'une "force du sang " pour expliquer ces épisodes spectaculaires ${ }^{66}$. La matière de La Force du sang, d'Alexandre Hardy, n'est-elle pas tirée des Nouvelles de Cervantès? On retrouve l'Espagne moderne et son obsession du sang et de l'honneur. Et l'on parlera bientôt de ces reconnaissances liées à la "race ", cachée, d'un homme - où comment, en un coup de théâtre, se révèle "noir" un homme qui se croyait blanc ou que l'on croyait tel.

Structurellement, on se découvre parents de la même manière que l'on se découvre de sang impur ou d'origine servile : c'est que l'on est dans de l'hypodescendance inversée, positive - parlons, encore une fois, d'hyperdescendance. On peut représenter les choses ainsi, mais au fond le schéma est le même que celui proposé pour l'hypodescendance (figure 1), sauf qu'il met en relation deux individus, $\mathrm{E}$ et $\mathrm{F}$ :

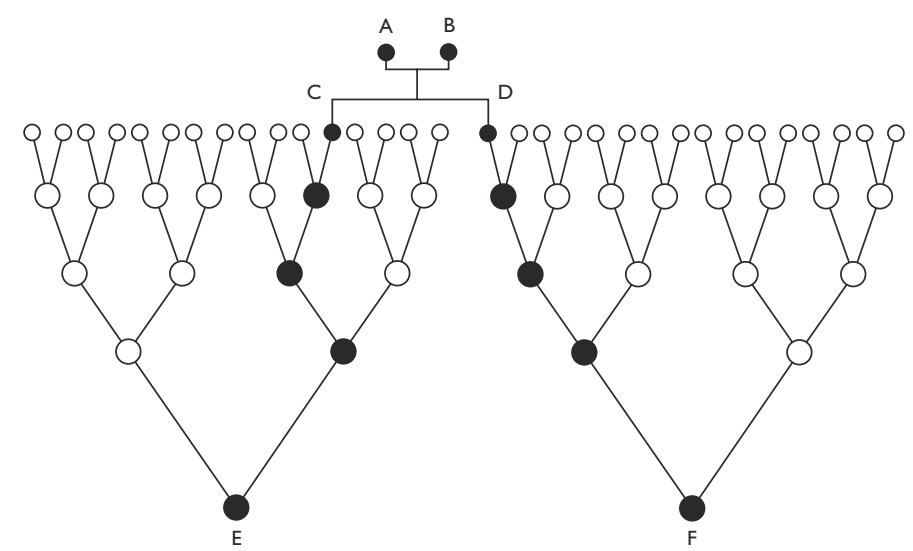

Fig. 3 “Ciel, mon cousin !” 
Sur ce schéma, on imagine que les individus $\mathrm{E}$ et $\mathrm{F}$ se découvrent parents : $\mathrm{C}$, un ancêtre de $\mathrm{E}$ (le père du père de la mère de son père), était l'enfant des individus $\mathrm{A}$ et $\mathrm{B}$, et $\mathrm{C}$ était donc le frère de $\mathrm{D}$; or $\mathrm{F}$ compte $\mathrm{D}$ parmi ses ancêtres ( $\mathrm{D}$ n'est-elle pas la mère de la mère de la mère de sa mère ?). $\mathrm{E}$ et $\mathrm{F}$ ont des arrière-arrière-arrière-grands-parents en commun : le couple A-B. À niveau social moyen, aujourd'hui, il nous paraît possible que deux individus qui seraient dans cette lointaine relation de parenté l'ignorent a priori. Qu'on la fasse reculer d'une ou deux générations, ou, en d'autres termes, que l'on considère qu'une ou deux générations de plus séparent $\mathrm{E}$ et $\mathrm{F}$, et cette méconnaissance deviendrait très plausible. Un seul ancêtre commun, en théorie du moins, suffit à rendre "parents » deux individus : l'inclusion dans une parenté commune suit le principe d'hyperdescendance. Reste que c'est plutôt de manière plaisante que l'on procédera à une telle «assignation "; et, en tout cas, c'est en cantonnant l'observation aux deux individus $\mathrm{C}$ et $\mathrm{D}$ que l'on pourra considérer qu'il s'agit là d'une assignation "au groupe supérieur" - car, a priori, l'observateur extérieur, lui, n'a pas de raison de juger la famille A-B comme supérieure à la sienne!

L'hyperdescendance connaît-elle de nombreuses réalisations historiques? Retournons au haut Moyen Âge, en Espagne wisigothique cette fois. Dans une logique certes persécutrice, mais encore confessionnelle et non raciale - dans une logique antijudaïque plutôt qu'antisémite, donc -, le concile de Tolède IV (633) considère comme nécessairement chrétien l'enfant d'un Juif et d'une chrétienne ou celui d'une Juive et d'un chrétien ${ }^{67}$ : désireux de faire reculer la "superstition judaïque » et de propager la « religion chrétienne ", le concile se montre, dans sa détermination de l'identité religieuse des enfants issus de mariage mixte, autrement plus inclusif (et donc adepte de l'hyperdescendance) que ne le seront l'Église et la société espagnoles presque mille ans plus tard.

Plus récemment, l'hyperdescendance est attestée dans le monde colonial: il semble que certaines catégories de la société brésilienne aient pratiqué une forme d'hyperdescendance, en encourageant dans une même lignée, plusieurs générations de suite, des mariages " mixtes " (entre homme blanc et femme noire ou l'inverse) : ce racisme "optimiste" entendait assurer le blanchissement progressif du pays, par absorption de la race noire dans la race blanche ${ }^{68}$. Des propositions similaires sont attestées dans les Antilles françaises au début du XIX ${ }^{\mathrm{e}}$ siècle ${ }^{69}$; mais l'idée est ici d' "absorber" les origines noires dans les origines blanches, plutôt que de doter les origines blanches d'une supériorité si marquée que les origines noires seraient négligeables. En Europe, les pratiques monarchiques contemporaines se rapprocheraient de l'hyperdescendance - mais simplement parce que le degré d'exigence formelle observé dans ces milieux 
nobiliaires a baissé. On voit que l'hyperdescendance, tout à fait pensable en théorie, trouve rarement des réalisations pratiques parfaites.

\section{Des cas divers, un principe commun}

La diversité des trois cas d'hypodescendance observés («Le pire l'emporte ", Limpieza de sangre, One-drop rule) est grande. Chacun a une finalité propre: application d'un statut et résolution de problèmes juridiques dans le premier cas, mise à l'écart voire expulsion pure et simple dans le deuxième cas, mise en ouvre d'une politique d'abord discriminatoire puis plus ambiguë, en même temps qu'obtention d'avantages idéologiques nets, dans le troisième cas.

En outre, si le fonctionnement structurel de l'hypodescendance est trois fois identique, l'ancrage à l'identité est, lui, variable : il s'agit simplement d'établir un statut, sans autres ambitions, pour «Le pire l'emporte». Pour la limpieza de sangre, théoriquement, une pratique religieuse et des traits culturels sont en jeu, mais ils sont en fait, comme on l'a vu, totalement vidés de contenu. Et pour la one-drop rule, la couleur est désignée, mais elle aussi de façon toute théorique, car on peut être phénoménologiquement blanc et "racialement» noir. Cette "noirceur invisible " ${ }^{70}$, forme extrême de la "pâleur noire " ${ }^{71}$, a d'ailleurs été le sujet central de mille et un romans nord-américains : on pense bien sûr à La Tache, roman récent de Philip Roth, dont le personnage principal, Coleman Silk, a longtemps pu cacher qu'il était "noir »" ${ }^{72}$ ou au célèbre Imitation of Life, de Fannie Hurst, avec le personnage de Peola, fort comparable ${ }^{73}$; mais bien d'autres œuvres ont fait de cette contradiction entre apparence et identité, aberration résultant de la one-drop rule, un élément décisif dans l'intrigue, qui tourne souvent autour de la révélation, en forme de reconnaissance, du fait que le personnage principal, en dépit des apparences, est «noir ${ }^{74}$. On voit que ce thème est d'une richesse insoupçonnée, et révèle la complexité de la situation et les difficultés qu'elle pose depuis plus d'un siècle à certaines consciences américaines ou même, plus récemment, occidentales.

Notons enfin que ce qui fait le pouvoir de chacun des groupes dirigeants considérés est très variable, et que les équilibres démographiques dans lesquels prend place le principe d'hypodescendance sont divers : sans doute une faible minorité de serfs face aux hommes libres, quelque position sociale que ces derniers occupent (des paysans modestes jusquaux grands nobles), dans l'Occident médiéval; dans l'Espagne moderne, après l'expulsion, une infime minorité d'hommes juifs ou considérés comme tels face aux chrétiens; et une minorité moins réduite de Noirs ou considérés comme tels face aux Blancs aux États-Unis. 
Par-delà cette diversité, quel est le sens de pareil comportement ? Il nous paraît clair qu'il trahit une forme d'angoisse : la transmission est inexorable, la tache (ou la macule) indélébile, le mal doit l'emporter sur le bien. Là est le principe commun à ces trois cas : quel que soit le vecteur de transmission, une goutte suffit à le propager, et rien - ni acculturation, ni conversion, ni apport de sang " pur »- ne peut plus l'empêcher de progresser : la métaphore de la contagion permet de bien comprendre ce que porte avec lui le concept. Une analyse serrée du thème du déclin et de la décadence des races dans le discours de l'extrême-droite nous mènerait sans doute à des idées similaires.

On retrouve chez les nazis cette même obsession de la dégénérescence, qui d'ailleurs ne concernait pas seulement le "sang juif »; la propagande nataliste entendait éviter que les "familles criminelles", réputées plus prolifiques, ne devinssent majoritaires dans la société. Des dessins publiés en 1937 dans un livre de propagande nous montrent comment, en 120 ans, risquent d'évoluer les proportions entre Vollwertige et Kriminelle: on passe d'une société composée de $50 \%$ de "familles de valeur" et d'autant de " familles criminelles » à une société où celles-ci ne représentent pas moins de $94 \%$ de la population, et celles-là seulement $6 \%$. Sur cinq colonnes disposées côte à côte, la partie occupée par les Kriminelle, qui font quatre enfants par famille (tout est là), augmente, et la partie qu'occupent les Vollwertige, qui ne font que deux enfants, diminue jusqu'à ne plus représenter, sur la colonne nach 120 Jahren ("après 120 ans »), qu'un petit histogramme (blanc) qui risque d'être submergé, en raison de la contagion criminelle sur le reste de la société ${ }^{75}$.

Or cette idée de l'inévitable propagation du mal constitue dans la pensée occidentale un véritable cliché, dont l'histoire est riche. La fin de l'époque médiévale, en particulier, cette époque inquiète et qui croit en la corruption, a abondamment réfléchi sur ce thème. Elle a utilisé à son propos, comme dans de nombreux autres cas, des images fortes et claires. On le voit à la lecture d'une prédication prononcée à Florence par Bernardin de Sienne en 1425. Citant Paul disant aux Corinthiens que les mauvais corrompent les bons, le franciscain exhorte son auditoire à s'écarter des mauvais. Citons-le longuement, car ses propos nous paraissent très significatifs :

"Ne converse avec aucun sodomite, car cela pourrait te saisir à ton tour ; de même, ne converse avec aucun hérétique, de peur qu'il ne te contamine; de même, ne converse avec aucun Juif, car, dans le Décret, cela t'est interdit ${ }^{76}$. [...] Une femme mauvaise, dans ton quartier, ne veuille pas d'elle dans ta maison! "Qui a un mauvais voisin a un mauvais matin"77. De même à l'école, un débauché sodomite contamine tous les autres. Prends-toi la bonne compagnie. Exemple : si tu prends des plumes d'aigle et que tu les mêles à la plume de la poule, celles de l'aigle consumeront les autres; de même la peau du loup consumera la peau de la brebis. Communément les cordes du 
luth et de la harpe sont faites d'intestins d'agneau ou de chevreau ; s'il y a mélange, les cordes d'entrailles de loup, jamais tu ne trouveras d'accord avec elles, et cela en raison de l'inimitié naturelle; le mauvais est toujours ennemi du bon. Le basilic ${ }^{78}$, avec sa vue, empoisonne l'homme; un mauvais regard tue l'âme. Saint Pierre apôtre, quand il fut avec les méchants, alors que Jésus-Christ était captif, le renia ${ }^{79}$, mais quand il partit d'eux, il retourna à pénitence ${ }^{80}$.

Dans ce discours, qui tourne autour de l'idée de la compagnie dont il faut ou non s'entourer, le prédicateur fait donc figurer, dans la liste des gens à éviter, les Juifs. Coïncidence parlante : ce passage illustre de façon significative la métaphore de la contagion, qui commande au modèle anxieux de l'hypodescendance, et il «naturalise » le phénomène en l'ancrant pratiquement dans les lois de la physique ou de la biologie, pour autant que Bernardin eût le concept de ces sciences. Il s'inscrit dans l'histoire de l'idée de corruption, depuis le traité De la génération et de la corruption d'Aristote jusqu’à l'époque contemporaine en passant peut-être par Bernardin et quelques autres prédicateurs et sûrement par Augustin, sans oublier l'étape fondamentale qu'est la modification que les évangiles font subir aux notions "vétérotestamentaires" de pureté et d'impureté ${ }^{81}$. L'idée paraît liée à celle que ce monde-ci est celui de la corruption. L'âme se corrompt dans le corps et de la même manière, dans le domaine de la science physique, les objets se corrompent. Plus trivialement, le thème de la "mauvaise compagnie », cher aux moralistes, des «Enseignements " de Louis IX à son fils ${ }^{82}$ au tableau de Jan Steen ${ }^{83}$, appartient à la même thématique.

Reste à comprendre quelle continuité existe entre ces trois cas d'hypodescendance : c'est poser là une question compliquée. On est manifestement face à des sociétés différentes mais liées entre elles et qui, se trouvant dans une configuration proche, formulent une solution identique. Mais de continuité substantielle, de celle qui existe quand un concept est consciemment transmis d'un univers culturel donné à un autre (quitte à ce qu'il change en partie de sens à l'occasion de la transmission), il ne peut sans doute guère être question ici : nulle continuité "de contenu" ne parait relier «Le pire l'emporte» et la one-drop rule via la limpieza ${ }^{84}$.

\section{Pour conclure: approches comparatistes}

Historiquement, on observe l'application du principe d'hypodescendance dans des sociétés «malades » de leur pureté, de leur propreté, jalouses de ce que chacun sait que l'on ne peut plus contrôler ni modifier: le passé. Quelles sont ces sociétés? Des sociétés profondément fragiles, sans doute, en crise ou au bord de la crise, en proie du moins à l'inquiétude ? La réponse semble évidemment positive. Il n'en est rien. La France du XII siècle, l'Espagne du XVI e et les États-Unis du XXe sont tout sauf des pays en déclin : tous 
trois sont au contraire des pays en plein essor, voire au mieux de leur histoire. La première, menée par un souverain respecté régnant sur un peuple très nombreux, est le royaume le plus puissant d'Occident; elle participe de manière décisive aux croisades et connaît un essor économique et démographique remarquable. La deuxième est au cœur d'une "économie-monde", avec sa flotte, ses explorations, ses colonies, son or ; elle fait basculer de la Méditerranée vers l'Atlantique l'équilibre des échanges, qui tourne à son profit. Quant aux troisièmes, ils sont au XIX et au XXe siècle la puissance montante, rivalisant avec les vieilles puissances européennes jusqu'à la Première Guerre mondiale, puis l'un des deux "grands", et aujourd'hui la seule "superpuissance", souvent capable d'imposer sa volonté et disposant de moyens considérables pour ce faire. Ce n'est donc pas dans un pays angoissé par son déclin que s'impose ce principe : paradoxalement, il survient dans des centres du monde, dans des lieux de pouvoir débordant de force. Là, le groupe dominant (précisons que sa domination est sociale et aussi numérique), qui voit peut-être les évolutions sociales comme une menace, se sent capable, dans tous les cas considérés, d'édicter une législation dont la première conséquence démographique sera de fermer le groupe et les modalités d'accès à celui-ci.

Pareille fermeture trahit-elle un sentiment de confiance en soi ou, au contraire, une forme de crispation, de crainte éprouvée par le groupe dominant face aux éléments qui, eux, connaîtraient une progression? La question est ouverte. Quoi qu'il en soit, on observe que ce groupe se sent, à un certain moment, porté à une attitude susceptible d'entraîner un affaiblissement démographique, tandis qu'il est d'autres cas où, au contraire, il juge nécessaire, pour ne pas disparaître, d'intégrer des individus (on a dit que la noblesse contemporaine était ainsi contrainte de s'amollir : par nécessité, les «mésalliances » d'antan sont mieux acceptées). Ces trois sociétés fortes et en croissance étaient confrontées plus que dans le passé à l'altérité et au changement : là réside peut-être une explication du paradoxe.

L'application de l'hypodescendance répond à un besoin de compter combien il y a de serfs, de Juifs, de Noirs; ou à un besoin de savoir qui est serf, juif, noir. Et l'on observe toujours la croyance selon laquelle l'hérédité transmet quelque chose d'indissoluble : c'est bien là le fondement du concept d'hypodescendance. Dans ce monde sans nuances, un seul ancêtre inférieur ("hypo-»), c'est-à-dire «mauvais» (un serf, un Juif, un Noir), suffit, de quelque côté qu'il vienne (ascendance matrilatérale ou patrilatérale) et fût-il minoritaire, à rendre Ego inférieur.

Proposons une présentation plus abstraite du système, en soulignant les règles fondamentales de son fonctionnement ${ }^{85}$. Disons pour commencer que l'hypodescendance repose, bien sûr, sur un principe d'inégalité (la 
hiérarchie et la dissymétrie entre les deux groupes sont explicites), et sur les règles de descendance (l'appartenance de l'individu a à voir avec celle de ses géniteurs) et d'identité (l'enfant de deux parents appartenant au groupe supérieur appartient au groupe supérieur, l'enfant de deux parents appartenant au groupe inférieur appartient au groupe inférieur). L'appartenance de l'individu au groupe est entière, en ce sens qu'elle ne connaît pas de demi-mesures (et il n'existe d'ailleurs, nécessairement, que deux groupes). On n'observe pas, contrairement au cas des colonies, de pleine application de "la règle de non retour", qui voudrait que seul l'enfant de deux individus du groupe $\mathrm{A}$ soit $\mathrm{A}$, et que seul l'enfant de deux individus du groupe B soit B. Au contraire, dans l'hypodescendance, s'il est vrai que seul l'enfant de deux individus du groupe supérieur (libre / chrétien / blanc) appartient au groupe supérieur, en revanche, l'enfant né d'une union "mixte " (une union contractée entre un individu appartenant au groupe supérieur et un individu appartenant au groupe inférieur) appartient pleinement au groupe inférieur. Il est aussi serf / juif / noir que l'un de ses deux parents l'est. L'hypodescendance est dissymétrique : elle applique la règle de non retour pour le groupe supérieur seulement, et parvient ainsi à maintenir une classification excluant tout métissage, c'est-à-dire toute reconnaissance d'une identité complexe.

L'hypodescendance est un principe efficace, car il permet une cohérence absolue. Il exprime le refus total de toute solution partielle, le refus des demi-appartenances et des appartenances mixtes ou plurielles - de peur, sans doute, de ne savoir que faire des individus qui pourraient revendiquer ces appartenances : les asservir, les expulser, les exterminer? Les accepter, les favoriser? C'est là une manière forte d'affirmer, en creux, l'identité du groupe dominant, par une définition, négative, du groupe inférieur : la construction de l'altérité joue, dans celle de l'identité, un rôle décisif.

Pour atteindre cet objectif, l'hypodescendance fait, à la réalité qu'elle s'attache à décrire et à ordonner, une grande violence : face à une société métisse et même face à des individus métis, car nés d'unions mixtes, elle entend nier l'existence d'identités métisses. Plus rare que la filiation unilinéaire, qui paraît constituer la référence dominante pour déterminer l'appartenance, le principe d'hypodescendance n'est évidemment pas une manière arbitraire ou idéologiquement "neutre " de régler un dilemme. L'hyperdescendance n'est en effet pas la norme: jamais ou presque «le meilleur ne l'emporte ", pour parodier l'adage de droit médiéval.

Une proposition, pour terminer: ce principe, quoiqu'il soit presque oublié, n'est pas absent des représentations communes. D'une certaine manière, fût-elle inconsciente, il continue de déterminer un certain nombre de réflexes d'assignation des individus à une identité stable. Aussi 
nous paraît-il utile de connaître son existence, son fonctionnement et ses "déclinaisons ", aujourd'hui que des questions comme celles du métissage, des appartenances et des identités, « ethniques » notamment, occupent une plus vaste place dans le débat public.

\author{
EA 3350, "Analyse comparée des pouvoirs" \\ Université de Marne-la-Vallée, Champs-sur-Marne \\ savy_pierre@yahoo.fr
}

MOTS CLÉS /KEYWORDS : filiation - hypodescendance/hypodescent - métissage/ racial mixing parentélkinship - racisme/racism - unions mixtes/mixed marriages.

\title{
NOTES
}

1. Voir notamment le "prélude théorique" de Jean-Luc Bonniol, La Couleur comme maléfice: une illustration créole de la généalogie des Blancs et des Noirs, Paris, Albin Michel, 1992: 19-46.

2. Pierre Bourdieu \& Loïc J. D. Wacquant, "Sur les ruses de la raison impérialiste", Actes de la recherche en sciences sociales, 1998, 121122 : 109-118, 112. Dans les premiers temps, ces enfants étaient pour la plupart de père blanc et de mère noire; Audrey Smedley (Race in North America: Origin and Evolution of a Worldview, Boulder, Westview Press, 1993: 141) évoque ainsi ce qu'elle appelle l' « hypodescent " pour désigner, après Marvin Harris (Patterns of Race in the Americas, New York, Walker, 1964), la règle selon laquelle « children were accorded the lower status of their mothers" (c'est nous qui soulignons). Mais c'est une acception plus large du terme que nous retenons ici, conformément à l'usage désormais dominant. Ce que désigne la notion de "groupe inférieur" reste entièrement à définir. Sur les termes techniques de la parenté, nous renvoyons une fois pour toutes au "Glossaire de la parenté", L'Homme, 2000, 154-155: Question de parenté: 721-732.

3. Une approche comparative de la question des races a déjà été proposée par Jean-Paul Zuñiga, "La voix du sang: du métis à l'idée de métissage en Amérique espagnole", Annales, Histoire, Sciences sociales, 1999, 54 (2) : 425452. Aux pages 450-451, l'auteur conclut ce rapprochement entre "pureté de sang en Castille et manière de concevoir la miscégénation américaine" sur l'idée qu'elles ont "des origines communes ", mais que "leur transposition terme à terme ne paraît pas soutenable ", en raison de la différence entre leurs idéaux et, surtout, de la valeur différente des notions de sang et de métissage dans les contextes consi- dérés : différence qui ne pourrait être dépassée que par un recours abusif et anachronique à la notion de "race". Nous tenterons infra de répondre à ces objections classiques et importantes, dont nous avons déjà discuté dans " "Les Juifs ont une queue." Sur un thème mineur de la construction de l'altérité juive", Revue des études juives, 2007, 166 (1) : 117-150.

4. Sur ce principe, voir Paul Viollet, Histoire du droit civil français accompagnée de notions de droit canonique et d'indications bibliographiques, Paris, L. Larose \& Forcel, 1893 [2 éd.] : 311-336 (et particulièrement pp. 319-320) ; François OlivierMartin, Histoire de la coutume de la prévôté et vicomté de Paris, Paris, E. Leroux, 1922 : I, 137 ; et Robert Boutruche, Seigneurie et féodalité. 2 : L'apogée (XI'-XIIr siècles), Paris, Aubier, 1970 : 6263. Pour la région parisienne, Guy Fourquin (Les Campagnes de la région parisienne à la fin $d u$ Moyen Âge, du milieu du XIIr siècle au début du $X V T^{e}$ siècle, Paris, Puf, 1964) offre une bonne mise au point (voir notamment «Les classes juridiques dans la paysannerie": 160-172). On dispose avec Pierre Bonnassie ("Liberté et servitude", in Jacques Le Goff \& Jean-Claude Schmitt, eds, Dictionnaire raisonné de l'Occident médiéval, Paris, Fayard, 1999: 595-609), d'une présentation synthétique de ce que désignent ces catégories, tandis que Michael M. Sheehan ("Theory and Practice: Marriage of the Unfree and the Poor in Medieval Society ", Mediaeval Studies, 1988, 50 : 457-487, repris in M. M. Sheehan, Marriage, Family and Law in Medieval Europe: Collected Studies, éd. James K. Farge, Toronto-Buffalo, University of Toronto Press, 1996 : 211-246) présente clairement la question du mariage des non-libres, et même celle de leur mariage avec un libre ("The Right to Marry": 227-236), mais n'envisage guère le sort des enfants nés de ces unions. 
5. Theodosiani Libri XVI cum constitutionibus sirmondianis, 1, éd. Theodor Mommsen, Berlin, 1905, 4, 8, 7 : 185, Interpretatio (texte présent dans certaines leçons manuscrites seulement): "Si quis in libertate positus ancillam sibi copulauerit in coniugium, si filii ex ipsis nati fuerint, origo matrem sequatur: simili modo, si liberta seruum duxerit maritum, agnatio seruum sequatur, quia ad inferiorem personam uadit origo". Voir aussi, dans Theodosiani..., op. cit., 5, 18, $1(5,10,1): 239-240$, et $9,9,1(9,6,1): 451-452$.

6. Voir Isidore de Séville, Étymologies, 9, 5, 18 : "Item liberi dicti, quia ex libero sunt matrimonio orti. Nam filii ex libero et ancilla servilis condicionis sunt. Semper enim qui nascitur deteriorem parentis statum sumit ». Gratien, 2, 32, 4, 15: "Semper enim qui nascitur deteriorem partem sumit ». Et voir encore Raban Maur, De rerum naturis (De universo), 7, 3. En outre, sur la servitude dans le droit canonique, voir John Gilchrist, «The Medieval Canon Law on Unfree Persons: Gratian and the Decretist Doctrines, c. 1141-1234", Studia Gratiana. Mélanges G. Fransen, 1976, 19: 271-301, notamment pp. 293-294.

7. Lex Ribuaria, Monumenta Germaniae historica, Legum, 1, Legum nationum Germanicarum, 3, 2, éd. Franz Beyerle, Hanovre, 1954 , p. 112, 61 (58), 11: "Si ecclesiasticus, Romanus vel regius homo ingenuam Ribvariam acciperit, aut si Romana vel regia seu tabularia ingenuum Ribvarium in matrimonium acciperit, generatio eorum semper ad inferiora declinentur [sic]». Voir aussi Leges Visigothorum, éd. Karl Zeumer, Monumenta Germaniae historica, Legum, 1, Legum nationum Germanicarum, 1, Hanovre-Leipzig, 1902, 3, $2: 135$. Sur la présence du principe dans les lois romano-barbares, voir Paul Allard, Les Origines du servage en France, Paris, Gabalda, 1913: 57.

8. Leges Burgundionum, éd. Ludwig Rudolf von Salis, Monumenta Germaniae historica, Legum, 1, Legum nationum Germanicarum, 2, 1, Hanovre, 1892, "Leges Burgundionum Lex Romana ", 37, 5: 156: "Inter ingenuum vero et ancillam, sive servum et ingenuam $[\ldots]$, et qui ex his nati fuerint, deteriorem lineam secuti dominis adquiruntur ».

9. Nivernais, Coutume de 1490, VIII, in François Ragueau, Glossaire du droit françois, Niort, L. Favre, 1882: 327-329. Une formule presque identique dans la coutume de 1534 (éd. 1610), VIII. Pour le Bourbonnais, art. 208 et 298; voir aussi l'art. 199. Ragueau renvoie aussi à une lettre d'Yves, évêque de Chartres, où il est question du mariage entre personnes de rang différent («entre hommes libres et serves, et réciproquement »), des cas où il convient de les conserver et de ceux au contraire où l'on peut les dissoudre (Lettres de saint Ives, évêque de Chartres, éd. Lucien Merlet, Chartres, E. Garnier, 1885, lettre 243, mais 242 dans la numérotation qu'utilise Ragueau, pp. 438-440 ; voir aussi la lettre 222, pp. 398-399; voir en outre Yves de Chartres, Tripartita, B, 15, 44). Sur tous ces aspects, il faut encore renvoyer à Paul Viollet, Histoire..., op. cit. : 319-320.

10. Voir Guy Fourquin, Les Campagnes..., op. cit. : 166-167.

11. Voir Les Établissements de saint Louis, éd. Paul Viollet, Paris, Librairie Rouard, 1883 : I, 42 et 78 , et III, 283. On trouve sous une forme assez détaillée le même principe de détermination par le statut de la mère, indépendamment du père, dans Philippe de Beaumanoir, Coutumes de Beauvaisis, éd. Amédée Salmon, Paris, 1900 : 2, XLV, 223-22, notamment 1434; et Nicolas Brussel qui cite les Établissements et évoque bien le cas d'un homme né de mère franche qui n'est pas serf même si son père l'est (Nouvel examen de l'usage général des fiefs en France pendant le XI, le XII, le XIII et le XIV siècles, pour servir à l'intelligence des plus anciens titres du domaine de la Couronne, Paris, 1727, 2 : 930). C'est ainsi que Marc Bloch oppose les deux principes, «le pire l'emporte » et la filiation maternelle, et observe que cette dernière thèse "paraît avoir généralement triomphé dans la littérature juridique" (Marc Bloch, "Liberté et servitude personnelles au Moyen Âge, particulièrement en France : contribution à une étude des classes", Anuario de Historia del Derecho Español, 5: 5-101, repris dans M. Bloch, Mélanges historiques, Paris, Sevpen, 1963 : I, 286-355, p. 293, note 3).

12. Antoine Furetière, "Libre", in Dictionnaire universel, Den Haag-Rotterdam, A. \& R. Leers, 1690, 2.

13. Décrétales , 4, 10, 1, «De natis ex libero uentre » : « Natus ex libera uel liberta liber est ».

14. Pour citer Laurent Boyer \& Henri Roland: «Pars viscerum matris / Dans le sein de la mère (Locutions latines du droit français, Paris, Litec, 1998 : 344). Voir également Laurent Boyer \& Henri Roland, Adages du droit français, Paris, Litec, 1999 (adage $\mathrm{n}^{\circ} 313$ : «Partus sequitur ventrem / La part suit le ventre [d'après Gaïus, Inst. I, 82] » : 622-625).

15. Voir Robert Fossier (Enfance de l'Europe, $X^{e}$-XII siècles. Aspects économiques et sociaux. 1 , L'homme et son espace, Paris, Puf, 1982: 579580) qui toutefois ignore «le pire l'emporte». 
16. Cf. Thomas d'Aquin : «Utrum filii debeant sequi conditionem patris»: "In quibusdam tamen terris quae iure ciuili non reguntur, partus sequitur deteriorem conditionem; ut si pater sit seruus, quamuis mater sit libera, erunt filii serui " (Scriptum super Sententiis, Parme, 1858, IV, 36, 4).

17. Pour citer Pierre Petot: « La règle, d'origine romaine, qui fait dépendre le statut de l'enfant de celui de la mère, ne s'introduit qu'au XIII ${ }^{e}$ siècle dans les régions coutumières françaises". ("Licence de mariage et formariage de serfs dans la coutume française du Moyen Âge ", Czasopismo prawno-historyczne. Annales d'histoire du droit de Poznan, 1949 : II, 199-208, 205).

18. Coutumes de la province et comté-pairie de la Marche, éd. Abdon-René Couturier de Fournoue, Clermont-Ferrand, 1744, article 154 : 104-105. Il faut pour cela qu'un homme tenant par ailleurs un héritage "serf ou mortaillable » ait, "l'espace de trente ans", tenu cet héritage franc.

19. Encyclopédie, ou Dictionnaire raisonné des sciences, des arts et des métiers, Paris, 1757 : VII, 172.

20. Sur le formariage, voir Pierre Petot, "Licence... », art. cit. ; et Michel Lebon, "Textes sur le formariage en Lorraine des origines au début du XIII" siècle", Annales de l'Est, 1951 : 53-66, qui n'envisage pas le cas du mariage entre serfs et libres, au motif que ce "cas est beaucoup plus rare et moins dangereux pour le seigneur » que les formariages par excellence, ceux que contractent des serfs de seigneuries différentes (p. 55).

21. Le Coutumier bourguignon glosé (Fin $d u$ $X I V^{v}$ siècle), éd. Michel Petitjean \& Marie-Louise Marchand, sous la dir. de Josette Metman, Paris, Éd. du CNRS, 1982 : 258-259: «Se uns homs de mainmorte prent femme franche, elle est de la condicion son mari, le mariage durant; et se elle muert en lieu du mari serf, le seigneur du mari serf aura la succession de la femme, supposé qu'il n'y ait hoir en celle; se homs se marie en lieu serf et prent femme serve, se il va demourer ou lieu serf et il advoue le seigneur du lieu serf, il est faiz serfs, ou se il ly demeure par an et par jour ou lieu serf, et se il muert, le seigneur a la succession, posé qu'il n'y ait hoir ; car homs frans se puet faire serf et advouer seigneur en servitute; mais homs serfs ne puet devenir frans, quelque part qu'il aille, senz faire desavoul au seigneur dessoubz qui il part". Voir aussi une idée semblable p. 46, les quatre cas envisagés p. 261 et des dispositions proches p. 282.

22. Antoine Loysel, Institutes coutumières, Paris, 1608, rééd. Institutes coutumières d'Antoine
Loysel... avec les notes d'Eusèbe de Laurière, éd. Charles Dupin, Eusèbe de Laurière \& Édouard Laboulaye, Paris, 1846, règle 43 : I, 59-60.

23. Il faut reprendre Antoine Loysel, Institutes..., op. cit. : 56 sqq. On lit successivement : "Par la plupart des coutumes la verge anoblit, et le ventre affranchit», "Naturellement les enfants nés hors mariage suivent la condition de la mère ", "En mariage légitime ils suivent la condition du père ", "Et en formariage le pire emporte le bon.»

24. Antoine Furetière, "Race", in Dictionnaire..., op. cit., 3.

25. Ainsi, dans la Castille du XV e siècle, est "roturier tout enfant né d'un père non noble, quelle que soit la condition de la mère " (MarieClaude Gerbet, La Noblesse dans le royaume de Castille: étude sur les structures sociales en Estrémadure de 1454 à 1516, Paris, Publications de la Sorbonne, 1979: 107).

26. Il existe sur ces thèmes une bibliographie immense. Voir Henri de Boulainvilliers, Essai sur la noblesse de France, Amsterdam, 1732, source particulièrement célèbre; et André Devyver, Le Sang épuré: les préjugés de race chez les gentilshommes français de l'Ancien Régime (1560-1720), Bruxelles, Éditions de l'Université, 1973.

27. Antoine Furetière, "Noble", Dictionnaire..., op. cit., 2.

28. Comme le rappelle justement Léopold Génicot: «En définitive, les textes ne connaissent qu'un élément permanent et consubstantiel au groupe: le sang" ("Noblesse», in Jacques Le Goff \& Jean-Claude Schmitt, eds, Dictionnaire..., op. cit. : 822).

29. Encore une fois, la bibliographie sur ces thèmes est abondante. Voir, outre André Devyver, Le Sang..., op. cit. , Andrew W. Lewis, Le Sang royal: la famille capétienne et l'État, France, $X^{e}-X I V^{v}$ siècle, Paris, Gallimard, 1986 ; et Le Sang au Moyen Âge, Montpellier, Université Paul-Valéry, 1999.

30. Suétone, Auguste, 40 : "Magni praeterea existimans sincerum atque ab omni colluuione peregrini ac seruilis sanguinis incorruptum seruare populum, et ciuitates Romanas parcissime dedit et manumittendi modum terminauit ».

31. Sur la notion, très discutée, de limpieza, voir Albert A. Sicroff, Les Controverses des statuts de "pureté de sang" en Espagne du XVe au XVII siècle, Paris, Didier, 1960 ; et Yosef Hayim Yerushalmi, «L'antisémitisme racial est-il apparu au XXe siècle? De la limpieza de sangre espagnole 
au nazisme : continuités et ruptures ", Esprit, 1993, $190:$ 5-35, repris dans Y. H. Yerushalmi, Sefardica, Essais sur l'histoire des Juifs, des marranes et des nouveaux chrétiens d'origine hispano-portugaise, Paris, Chandeigne, 1998 : 255-292.

32. Albert A. Sicroff, Les Controverses..., op. cit. : 25.

33. Comme le souligne Joseph Pérez ( La pureté de sang dans l'Espagne du XVI e siècle", in Robert Sauzet, ed., Les Frontières religieuses de l'Europe du XVe au XVIT siècle, Paris, Vrin, 1992 : 109-117) qui affirme le caractère strictement sociologique de la pureté de sang et tend à limiter la diffusion et l'importance des «statuts de pureté». Sur la place de la pureté dans l'Inquisition, voir les positions nuancées de Jean-Pierre Dedieu, L'Administration de la foi: l'Inquisition de Tolède, XVI'-XVIIT siècle, Madrid, Casa de Velásquez, 1989: 332-346.

34. Ferrand Mexía, Nobiliario vero, Sevilla, 1492.

35. Nous empruntons ici à Adeline Rucquoi, "Être noble en Espagne aux XIV ${ }^{\mathrm{e}}-\mathrm{XVI}^{\mathrm{e}}$ siècles", in Gerhard Oexle Otto \& Werner Paravicini, eds, Nobilitas: Funktion und Repräsentation des Adels in Alteuropa, Göttingen, Vandenhoeck \& Ruprecht, 1997 : 273-298; Adeline Rucquoi, "Mancilla y limpieza : la obsesión por el pecado en Castilla a fines del siglo XV», in Os "últimos fins" na cultura ibérica dos séculos XV-XVIII, colloque de Porto, 19-21 octobre 1995, Porto, Instituto de cultura portuguesa, 1997 : 113-135; et Adeline Rucquoi, "Noblesse des conversos?", "Qu'un sang impur... " Les conversos et le pouvoir en Espagne à la fin du Moyen Âge, colloque d'Aixen-Provence, 18-20 novembre 1993, Aix-enProvence, Publications de l'université de Provence, $1997: 89-108$.

36. Nous avons évoqué supra l'Essai de Boulainvilliers; voir ce qui est dit de la «guerre des races" dans Michel Foucault, Il faut défendre la société, Cours au Collège de France (1975-1976), Paris, Gallimard-Le Seuil, 1997.

37. Madison Grant, The Passing of the Great Race: Or the Racial Basis of European History, New York, Charles Scribner's Sons, 1916.

38. Sur la race aux États-Unis, dans une bibliographie bien sûr considérable, voir Marvin Harris, Patterns..., op. cit.; Joel Williamson, New People: Miscegenation and Mulattoes in the United States, New York, Free Press, 1980, notamment l'introduction : 1-4 (qui s'ancre dans l'actualité de la question et insiste beaucoup sur la spécificité américaine - «Our paradox is unique", p. 2) ; Floyd James Davis, Who is Black? One Nation's Definition, University Park, The Pennsylviana State University Press, 1991 ; et récemment Lawrence A. Hirschfeld, qui, dans une approche empirique relevant plutôt des sciences cognitives, porte sur l'apprentissage des notions par les enfants ("La règle de la goutte de sang ou comment l'idée de race vient aux enfants ", L'Homme, 1999, 150 : 15-39).

39. Audrey Smedley (Race..., op. cit. : 246) évoque "the value of "pure" white "blood" that became such an obsession in Anglo-Saxon America ".

40. Paul Schor, "Statistiques de la population et politique des catégories aux États-Unis au XIX ${ }^{\mathrm{e}}$ siècle: théories raciales et questions de population dans le recensement ", Annales de démographie historique, 2003, 1 : 11-12.

41. Sur cet aspect, voir Loïc J. D. Wacquant, «From Slavery to Mass Incarceration: Notes for Rethinking the "Race Question" in the United States", New Left Review, 2002, 13 : 41-60 et 45, et surtout Barbara Jeanne Fields, "Slavery, Race and Ideology in the United States of America ", New Left Review, 1990, 181, 1 (13) : 114 .

42. Sur cet épisode, voir Jean-Paul Zuñiga, "La voix... ", art. cit. : 425-426.

43. Sur ce point, voir le chapitre 6, de Floyd James Davis, Who..., op. cit.: 123-139; et Christine B. Hickman ("The Devil and the One Drop Rule: Racial Categories, African Americans, and the U. S. Census", Michigan Law Review, 1997, 95 (5) : 1161-1276) qui montre que cette question est encore vivante (puisqu'il s'agit aussi pour l'auteur de faire des propositions sur le recensement américain) et que nombreuses demeurent les ambiguïtés visà-vis de la "one-drop rule", née dans des conditions détestables (le "Devil» du titre) mais qui aurait eu, en termes historiques et politiques, des effets positifs.

44. Un débat opposa divers spécialistes de ces questions (voir notamment Michael Hanchard, "Black Cinderella? Race and the Public Sphere in Brazil ", Public Culture, 1994, 7 : 165-185). Sur le lexique des catégories, voir : Magnus Mörner, Race Mixture in the History of Latin America, Boston, Little Brown \& $\mathrm{C}^{\circ}, 1967$, où figure un incroyable tableau avec les noms des différentes catégories métisses; Manuel Alvar, Léxico del mestizaje en Hispanoamérica, Madrid, Ediciones cultura hispanica, Instituto de cooperación iberoamericana, 1987, lexique d'une richesse étonnante puisque, de l' «ahí» au 
«zambo », il faut à l'auteur plus d'une centaine de pages pour présenter et définir tous les termes qualifiant les métis; et, concernant le domaine français, le non moins étonnant volume de Médéri Louis-Élie Moreau de SaintMéry, Description topographique, physique, civile, politique et historique de la partie française de l'Isle Saint-Domingue, Philadelphia, 1797, en particulier aux pages 71-99.

45. Jean-Luc Bonniol, La Couleur..., op. cit. : 66.

46. Sur ces efforts, voir Paul Freedman, "Seigneurie et paysannerie au Moyen Âge: un retrait de l'historiographie américaine", Histoire et sociétés rurales, 2000, 14 : 153-168.

47. Sur le désir de protéger la "pureté généalogique » de la classe des prêtres, voir notamment Christine E. Hayes, Gentile Impurities and Jewish Identities: Intermarriage and Conversion from the Bible to the Talmud, Oxford-New York, Oxford University Press, 2002: 58-59. Voir aussi Talmud de Babylone, Bava Batra, 109b, qui exprime fortement la dimension patrilinéaire du judaïsme.

48. Sur tous ces aspects, sur lesquels il existe une abondante littérature, voir notamment Shaye J. D. Cohen, "The Origins of the Matrilineal Principle in Rabbinic Law ", Association for Jewish Studies Review, 1985, 10 : 1953, repris sous le titre "The Matrilineal Principle" dans S. J. D. Cohen, The Beginnings of Jewishness: Boundaries, Varieties, Uncertainties, Berkeley, University of California Press, 1999 : 263-307.

49. Cas célèbre grâce à Bronislaw Kaspar Malinowski, The Sexual Life of Savages in North Western Melanesia: An Ethnographic Account of Courtship, Marriage, and Family Life Among the Natives of the Trobriand Islands, British New Guinea, London, G. Routledge \& Sons, 1929. [Trad. franç. : La Vie sexuelle des sauvages $d u$ Nord-Ouest de la Mélanésie: description ethnographique des démarches amoureuses, du mariage et de la vie de famille des indigènes des îles Trobriand, Nouvelle-Guinée, Paris, Payot \& Rivages, 2000.]

50. Antoine Furetière, "Noble", in Dictionnaire..., op. cit., 2.

51. Talmud de Babylone, Qiddouchin, 68b, sur Deutéronome, 7, 3-4.

52. Sur ce point, voir Riccardo Di Segni, «Il padre assente. La trasmissione matrilineare dell'appartenenza all'ebraismo", Quaderni storici, 1989, 70 : 143-204.
53. Voir Bryan Mark Rigg, Hitler's Jewish Soldiers: The Untold Story of Nazi Racial Laws and Men of Jewish Descent in the German Military, Lawrence, University Press of Kansas, 2002. [Trad. franç. : La Tragédie des soldats juifs d'Hitler, Paris, de Fallois, 2003.]

54. Voir Raul Hilberg, The Destruction of the European Jews, Chicago, Quadrangle Books, 1961. [Trad. franç. : La Destruction des Juifs d'Europe, Paris, Fayard, 1988 : 61-64.]

55. Voir Raul Hilberg, La Destruction..., op. cit. : 67-68 et, p. 74, le résumé de la législation; Michael Burleigh \& Wolfgang Wippermann, The Racial State: Germany 1933-1945, Cambridge, Cambridge University Press, 1991, rééd. 1992, p. 45 ; ainsi que Mark Bryan Rigg (La Tragédie..., op. cit.), qui, avant de traiter spécifiquement la question des soldats juifs, aborde ce point : 27-88.

56. «Provvedimenti per la difesa della razza italiana", décret-loi royal du 17 novembre 1938, « Capo II ». Sur les Juifs en Italie pendant le fascisme, voir encore Renzo De Felice, Storia degli ebrei italiani sotto il fascismo, Turino, Einaudi, 1961.

57. «Loi du 3 octobre 1940 portant statut des Juifs », Journal officiel, 18 octobre $1940: 5323$, article premier.

58. "Loi du 2 juin 1941 remplaçant la loi du 3 octobre 1940 portant statut des Juifs", Journal officiel, 14 juin $1941: 2475$.

59. Michael Burleigh \& Wolfgang Wippermann, The Racial..., op. cit. : 47.

60. Sur ces aspects, voir Saul Friedlander, L'Allemagne nazie et les Juifs. 1, Les années de persécution 1933-1939, Paris, Le Seuil, 1997 : 200 ; et Zeev Sternhell, "Anthropologie et politique: les avatars du darwinisme social au tournant du siècle ", in L'Allemagne nazie et le génocide juif, Paris, Gallimard-Le Seuil, 1985 : 53-75.

61. Voir Henry Méchoulan, Le Sang de l'autre ou l'honneur de Dieu: Indiens, juifs, morisques dans l'Espagne du Siècle d'Or, Paris, Fayard, 1979.

62. Sur ces aspects, voir Paul Julian Weindling, Health, Race, and German Politics between National Unification and Nazism, 1870-1945, Cambridge-New York, Cambridge University Press, 1989, rééd. Cambridge-New York, 1991, en particulier, «Nazi racial hygiene » : 489-564.

63. Voir supra sur la prise en considération, par les judaïsmes non orthodoxes, de l'ascendance juive patrilatérale. 
64. L'anagnorisis se définit simplement comme le passage de l'ignorance à la connaissance. Voir Aristote, Poétique, 6, 11, 14 et 16.

65. Denis Diderot, Le Fils naturel, ou Les épreuves de la vertu, Amsterdam, 1757, dans Diderot, Euvres complètes, 10, éd. Jacques Chouillet \& Anne-Marie Chouillet, Paris, Hermann, 1980: 13-81. La reconnaissance intervient dans la dernière scène de la pièce $(\mathrm{V}$, 5, p. 78) : "Mon fils, voilà ta sœur... Ma fille voilà ton frère...» Une didascalie précise même: "Dorval et Rosalie se regardent, tombent dans les bras l'un de l'autre ".

66. Voir Alexandre Hardy, La Force du sang, Paris, 1625, dans Théâtre du XVIT siècle, éd. Jacques Scherer, Paris, Gallimard, 1975: 131-184; et Clifton Cherpack, The Call of Blood in French Classical Tragedy, Baltimore, Johns Hopkins Press, 1958. Sur la reconnaissance, voir en outre Terence Cave, Recognition: A Study in Poetics, Oxford, Clarendon Press / New York, Oxford University Press, 1988; Umberto Eco, «L'agnizione: appunti per una tipologia del riconoscimento", dans U. Eco, $I l$ superuomo di massa, Milano, Bompiani, 1978 [trad. franç. : "L'agnition: notes pour une typologie de la reconnaissance", dans U. Eco, De Superman au surhomme, Paris, Grasset, 1993: 29-38 ]; sans oublier Paul Ricœur, Parcours de la reconnaissance: trois études, Paris, Stock, 2004, et Françoise Heulot-Petit \& Lise Michel, eds, La Reconnaissance dramatique, colloque d'Arras, 7-8 avril 2005, actes à paraître à Arras, 2007.

67. Voir le canon 63 du concile. Il s'agit d'abord des "Iudaei qui christianas mulieres in coniugio habent». On lit: «Filii autem qui ex talibus nati exsistunt, fidem atque condicionem matri sequantur». Mais le texte considère aussitôt l'autre cas, celui des enfants d'une juive et d'un chrétien : "Similiter et ii qui procreati sunt de infidelibus mulieribus et fidelibus uiris christianam sequantur religionem, non iudaicam superstitionem». Voir Bruno Dumézil, Les Racines chrétiennes de l'Europe: conversion et liberté dans les royaumes barbares ( $V^{e}$-VIII siècle), Paris, Fayard, 2005 : 672.

68. Voir Thomas Elliott Skidmore, Black into White: Race and Nationality in Brazilian Thought, New York, Oxford University Press, 1974, rééd. Durham-London, Duke University Press, 1993: 64-69.

69. Voir Jean-Luc Bonniol, La Couleur..., op. cit. : 79-83, qui présente l'idée d'un «blanchissement " sur six générations.
70. «Invisible blackness » dans le titre de Frank W. Sweet, Legal History of the Color Line: The Notion of Invisible Blackness, Palm Coast, Backintyme, 2005.

71. Voir Colette Pétonnet, «La pâleur noire: couleur et culture aux États-Unis ", L'Homme, 1986, 97-98: L'anthropologie: état des lieux: 171-188.

72. Philip Roth, The Human Stain, Boston, Houghton Mifflin, 2000. [Trad. franç. : La Tache, Paris, Gallimard, 2002.]

73. Fanny Hurst, Imitation of Life, New YorkLondon, Harper \& Brothers, 1933, maintes fois réédité depuis (sans parler des deux adaptations cinématographiques) et pour lequel on dispose même de traductions françaises (depuis Mirage de la vie, Paris, Plon, 1935) et d'une édition scientifique américaine (Fanny Hurst, Imitation of Life, éd. Daniel Itzkovitz, DurhamLondon, Duke University Press, 2004); outre cette édition, voir Lawrence A. Hirschfeld, « La règle...", op. cit. : 18-19.

74. C'est le cas (sans aucune prétention de notre part à l'exhaustivité) de Mark Twain, The Tragedy of Pudd'nhead Wilson, Hartford, American Pub. Co 1894 ; Charles Waddell Chesnutt, The House Behind the Cedars, Boston-New York, Houghton Mifflin, 1900; Nella Larsen, Passing, New York-London, A. A. Knopf, 1929; Lewis Sinclair, Kingsblood Royal, New York, Radom House, 1947. Sur le "passing", voir l'édition critique du livre de Nella Larsen (Larsen Nella, Passing: Authoritative Text, Backgrounds and Contexts, About Nella Larsen, Criticism, éd. Carla Caplan, New York, W. W. Norton \& Co , 2006).

75. Illustration tirée de Helmut Otto, Volk in Gefahr, München, Lehmann, 1937, et reproduite dans Michael Burleigh \& Wolfgang Wippermann, The Racial..., op. cit. : 171; voir aussi p. 169. Sur le caractère obsessionnel et «psychotique » de l'antisémitisme, voir aussi Saul Friedlander, L'Antisémitisme nazi : histoire d'une psychose collective, Paris, Le Seuil, 1971, notamment "La solution finale": 174-202. La même angoisse d'une transmission inexorable est soulignée par Henry Méchoulan, Le Sang..., op. cit. : 128, qui établit que l'Espagne connaissait à propos de la contagion juive une "obsédante inquiétude", liée entre autres à une "hantise virginale» (p. 142; voir les pages 119-143).

76. Sans doute Gratien, 2, 28, 1, 10 et 11.

77. Bernardin cite ici un proverbe populaire, encore attesté aujourd'hui en italien, et que n’ignorait pas la culture savante médiévale. 
78. Animal merveilleux auquel on prêtait, au Moyen Âge, la capacité d'empoisonner par le regard.

79. Le reniement de Pierre est dans Jean, 18, 15-27 ; Luc, 22, 54-62 et Marc, 14, 66-72.

80. Bernardin de Sienne, Le prediche volgariQuaresimale del 1425, éd. Ciro Cannarozzi, 2 , Firenze, 1940, prédication 21, "Come dei usare con le buone compagnie", pp. 16-17.

81. Voir Jean-Paul Zuñiga, "La voix...", art. cit. : 429-430.

82. Cf. Joinville: "[...] fui et eschieve la compaingnie des mauvez " (Vie de saint Louis, éd. Jacques Monfrin, Paris, Dunod, 1995, 745 : 59).

83. Steen Jan Havicksz, La Mauvaise compagnie, peinture à l'huile sur bois, vers 1650 1675, musée du Louvre, Paris.

84. La question est fort débattue pour les rapports de la limpieza de sangre avec l'antisémitisme de l'Allemagne nazie. Contre un Yerushalmi que l'on présente souvent comme un tenant radical du continuisme dans l'histoire de l'antisémitisme, mais dont les positions nous semblent en fait assez modérées, certains croient en une vraie continuité entre limpieza et lois racistes de l'Allemagne : voir Cecil Roth, $A$ History of the Marranos, Philadelphia, Jewish Publication of Society of America, 1941 [trad. franç. : Histoire des marranes, Paris, Liana Levi, 1990, rééd. 2002] ; Benzion Netanyahu, The Marranos of Spain from the late 14th to the early 16th Century According to Contemporary Hebrew Sources, New York, American Academy for Jewish Research, 1966, 3e éd. Ithaca, Cornell University Press, 1999; et Benzion Netanyahu, Toward the Inquisition: Essays on Jewish and Converso History in Late Medieval Spain, Ithaca, Cornell University Press, 1997.

85. Nous nous inspirons ici d'une démarche présente dans les travaux des anthropologues ayant étudié la théorisation du métissage dans les colonies, en particulier Jean-Luc Bonniol, La Couleur..., op. cit., et Pierre Crépeau, Classifications raciales populaires et métissage: essai d'anthropologie cognitive, Sainte-Marie, Centre de recherches caraïbes, 1972.

RÉSUMÉ/ABSTRACT

Pierre Savy, Transmission, identité, corruption: réflexions sur trois cas d'hypodescendance. - Dans trois contextes différents, une forme de filiation structurellement identique fut mise en œuvre: l'Occident médiéval avec le principe selon lequel «le pire l'emporte", l'Espagne moderne avec la limpieza de sangre et les États-Unis du XXe siècle avec la one-drop rule pratiquèrent l'« hypodescendance ", c'est-à-dire l'assignation des enfants d'une union mixte au groupe inférieur. Ce système original, opposé à la filiation unilinéaire et refusant toute identité complexe telle que le métissage en propose, opère un classement différent de celui proposé par les théories racistes du $\mathrm{XX}^{\mathrm{e}}$ siècle. Si ces cas d'hypodescendance (plus fréquents que ceux d'hyperdescendance) different entre eux par leur finalité et leur lien avec l'identité, ils traduisent tous le même besoin de classer et la même angoisse face à la propagation du «mal». Enfin, s'il n'y a guère de continuité entre eux, on observe qu'ils appartiennent tous à l'histoire de pays ou de régions du monde en plein essor et confrontés à l'altérité et au changement.
Pierre Savy, Inheritance, Identity, Corruption: Thoughts About Three Cases of Hypodescent. In three different contexts, a structurally identical form of descent came into use : Europe of the Middle Ages with the principle that «the worst wins "; modern Spain with limpieza de sangre; and the $20^{\text {th }}$ century United States with the "one-drop rule». Hypodescent, which occurs more frequently than hyperdescent, assigns the children of a mixed marriage to the subordinate group. This system contrasts with unilineal descent and rejects complex senses of identity such as mulatto or mestizo. Its classification differs from that of $20^{\text {th }}$ century racist theories. Even though cases of hypodescent differ from each other as to their finality and sense of identity, they all reflect the same need to classify and the same anxiety about the breeding of « evil». Despite the slight continuity between them, they all belong to the history of strongly growing countries or regions that had to cope with change and otherness. 